\title{
Evaluation of the osteogenic potential of rat adipose-derived stem cells with different polycaprolactone/alginate-based nanofibrous scaffolds: an in vitro study
}

\author{
Eman Hany ${ }^{1}$, Sarah Yahia ${ }^{2}$, Mahmoud Fathy Elsherbeny ${ }^{3}$, Nagla Mahmoud Salama ${ }^{3}$, \\ Islam Mohammed Ateia ${ }^{4}$, Noha Tharwat Abou El-Khier ${ }^{5}$, Ibrahim El-Sherbiny ${ }^{2}$, \\ Mazen Tharwat Abou Elkhier ${ }^{1}$
}

${ }^{1}$ Oral Biology Department, Faculty of Dentistry, Mansoura University, Mansoura, Egypt; ${ }^{2}$ Nanomedicine Lab, Center of Material Science, University of Science and Technology, Zewail City of Science and Technology, Giza, Egypt; ${ }^{3}$ Oral Pathology Department, ${ }^{4}$ Oral Medicine and Periodontology Department, Faculty of Dentistry, Mansoura University, Mansoura, Egypt; ${ }^{5}$ Medical Microbiology and Immunology Department, Faculty of Medicine, Mansoura University, Mansoura, Egypt

Contributions: (I) Conception and design: MT Abou Elkhier, E Hany; (II) Administrative support: NT Abou El-Khier; (III) Provision of study materials: S Yahia, I El-Sherbiny; (IV) Collection and assembly of data: E Hany, MF Elsherbeny; (V) Data analysis and interpretation: NT Abou ElKhier, NM Salama, IM Ateia; (VI) Manuscript writing: All authors; (VII) Final approval of manuscript: All authors.

Correspondence to: Eman Hany, PhD. Department of Oral Biology, Faculty of Dentistry, Mansoura University, Mansoura, Egypt. Email: eman_haney@mans.edu.eg.

Background: Bone tissue engineering is a widely growing field that requires the combination of cells, scaffolds and signaling molecules. Adipose derived stem cells (ADSCs) are an accessible and abundant source of mesenchymal stem cells with high plasticity. Polycaprolactone/alginate (PCL/Alg) composite scaffolds have been used in bone regeneration and nano-hydroxyapatite (n-HA) is used as a reinforcing, osteoconductive component in scaffold fabrication. This study was conducted to assess the ability of three different PCL/Alg based scaffolds to induce osteogenic differentiation of ADSCs and to compare between them.

Methods: The study comprised 5 groups; negative control group with ADSCs cultured in complete culture media, positive control group with ADSCs cultured in osteogenic differentiation media, and 3 experimental groups with ADSCs seeded onto 3 scaffolds: S1 (PCL/Alg), S2 (PCL/Alg/Ca) and S3 (PCL/Alg/Ca/n-HA) respectively and cultured in osteogenic media. Mineralization and gene expression were assessed by Alizarin red S (ARS) staining and real time quantitative polymerase chain reaction (RT-qPCR). Evaluation was done at 7,14 and 21 days.

Results: ARS staining reflected a time dependent increase through days 7, 14 and 21, with S3 (PCL/Alg/ $\mathrm{Ca} / \mathrm{n}-\mathrm{HA})$ group showing the highest mineralization levels. RT-qPCR detected upregulation of $A L P$ gene expression at day 7 and decline thereafter. S2 (PCL/Alg/Ca) and S3 (PCL/Alg/Ca/n-HA) groups showed significantly higher gene expression levels than S1 (PCL/Alg).

Conclusions: ADSCs and PCL/Alg-based scaffolds compose a good tissue engineering complex for bone regeneration. Addition of $\mathrm{n}-\mathrm{HA}$ to $\mathrm{PCL} / \mathrm{Alg}$ scaffolds and crosslinking with $\mathrm{CaCl} 2$ efficiently improve the osteogenic potential of ADSCs.

Keywords: Adipose-derived mesenchymal stem cells; alginate; hydroxyapatite; polycaprolactone (PCL); tissue scaffolds

Received: 25 March 2020; Accepted: 22 July 2020; Published: 07 August 2020.

doi: $10.21037 /$ sci-2020-015

View this article at: http://dx.doi.org/10.21037/sci-2020-015 


\section{Introduction}

Bone damage due to diseases, fractures, or tumors is a major problem. Regeneration of large bony defects by autogenic or allogenic grafts has been implicated but with many restrictions due to complicated surgical procedures with accompanied postoperative pain, limitation of bone quantity, donor site morbidity, immune rejection and high risk of infection (1).

Alternatively, tissue engineering has been recently used in bone and cartilage regeneration by expanding stem cells in vitro, seeding them onto scaffolds and implanting them in vivo in the defect site (2).

Mesenchymal stem cells are a heterogenous population of clonogenic cells with fibroblast like morphology that can adhere to plastic walls and differentiate into osteogenic, chondrogenic and adipogenic cells (3). Mesenchymal adipose stem cells are becoming an attractive source of stem cells in regenerative therapy because of their abundance and ease of harvest and cultivation (4). Adipose derived stem cells (ADSCs) have also shown ability to differentiate into ectodermal and endodermal lineages besides their own mesodermal lineage when stimulated under suitable culture conditions (5).

Scaffolds simulate stem cell niches aiding in their proliferation and differentiation and helps in alveolar bone formation $(6,7)$. An ideal scaffold should be biocompatible, biodegradable, allows cell attachment and interaction, and with suitable porosity (8).

Polycaprolactone (PCL) is a biocompatible, biodegradable, polyester material with good mechanical properties that has been used in multiple applications and as a scaffold for tissue engineering (9), but scaffolds made of synthetic materials only, are unable to achieve proper cell attachment due to their high hydrophobicity. Natural biological materials, on the other hand, are good means for tissue and organ regeneration (10).

Alginate is a natural hydrogel that is widely implicated in tissue engineering owing to its biocompatibility, hydrophilicity, ease of gelation and structural similarity to extracellular matrix (11), however its poor mechanical properties and difficulties in fabrication of 3D scaffolds are obstacles that limit its usage in hard tissue engineering (12).

To overcome these limitations, hydrogels are incorporated into other synthetic biomaterials so as to get the biological advantages of hydrogels and the good mechanical properties of synthetic materials (10). Alginates have been combined with many synthetic materials including PCL, to form composite organic/inorganic scaffolds for hard tissue engineering $(12,13)$.

Nano-hydroxyapatite (n-HA) and collagen form the main constituents of bone (14). Hydroxyapatite is an inorganic ceramic used as a reinforcement particle in scaffold fabrication. It was found to be highly bioactive and to possess good osteoconductive properties (15). Moreover, ceramic scaffolds with nanoscale-sized particles are known to possess better mechanical properties (16). But despite strength, HA is highly brittle and hard to shape and that is why it is usually blended with other materials to form composite scaffolds (17).

Alginates together with HA can form porous, threedimensional, biodegradable, osteoconductive scaffolds that make a solid system for natural bone regeneration $(18,19)$. Hydroxyapatite has also been used in combination with PCL as scaffolds for bone tissue engineering $(17,20)$.

Combination of PCL with alginate and n-HA in a single scaffold hasn't been introduced so far and comparison between different scaffolds can help establish a novel hybrid scaffold that may aid in bone tissue engineering field.

So, this study was conducted to test the effect of three different PCL/alginate (PCL/Alg) based scaffolds on the osteogenic differentiation capacity of adipose derived mesenchymal stem cells and to compare between them.

\section{Methods}

All procedures involving animals were approved by the ethical committee of faculty of dentistry, Mansoura University with code number: A13091019, in compliance with Mansoura University guidelines for the care and use of animals.

\section{Materials}

PCL, N,N-dimethylformamide (DMF), dichloromethane (DCM), sodium alginate and calcium chloride were purchased from Sigma-Aldrich (China and Germany). Calcium hydroxide powder (99\%) was purchased from Carl Roth (Karlsruhe, Germany). Acetic acid (99.8\%), sodium hydroxide (96\%) and absolute ethanol (99.8\%) were purchased from Sigma-Aldrich (St. Louis, MO, USA). Orthophosphoric acid (98\%) was obtained from BDH Chemicals Ltd. (Poole, England).

Type 1 Collagenase enzyme and Dulbecco's Modified Eagle Medium/Nutrient Mixture F-12 (DMEM/F12) were purchased from Lonza (Basel, Switzerland); 10\% fetal 
bovine serum (FBS) was obtained from Sigma Aldrich (St. Louis, MO, USA). Osteogenic medium Cat No. CCM009 was purchased from R\&D systems, Inc. (Minneapolis, MN, USA). Alizarin red S (ARS) stain was obtained from Fischer scientific (Bishop Meadow Road, Loughborough, UK). Fluorescent-labeled antibodies were obtained from Abcam (Cambridge, UK).

\section{Scaffold fabrication}

\section{Fabrication of the middle nanofiber PCL layer (PCL NFs)}

PCL layer (PCL NFs) was constructed using electrospinning technique (NANON-01A electrospinning system, MECC Co., LTD) and characterized physically and mechanically as described in our previous study (21). In brief, PCL solution was made by dissolving the polymer $10 \mathrm{wt} \%$ in a mixture of DCM and DMF (60: $40 \mathrm{wt} \%$ ). Then the solution was fed in a plastic syringe with diameter $13.1 \mathrm{~mm}$. Nanofibers were collected by adjusting the voltage, flow rate, and the distance between the syringe tip and the ground receiver at $26 \mathrm{kV}, 3.5 \mathrm{~mL} / \mathrm{hour}$ and $15 \mathrm{~cm}$, respectively.

\section{Preparation of nano hydroxyapatite (n-HA)}

The powder was synthesized via in-situ co-precipitation method as described in our previous paper (22). The reaction was done by dripping $\mathrm{H}_{3} \mathrm{PO}_{4}(10 \%$ w/v) solution into ethanolic solution of $\mathrm{Ca}(\mathrm{OH})_{2}(4 \%)$ with continuous ultrasonication then adjusting the final $\mathrm{PH}$ to 10 by using $\mathrm{NaOH}$ solution and stirring the mixture for 24 hours. The obtained slurry was kept without stirring for another 24 hours. Lastly, the filtered precipitate was washed by deionized water and aged at $50^{\circ} \mathrm{C}$ in a vacuum oven.

\section{Incorporation of n-HA in NFs sheets}

n-HA particles were mixed with sodium alginate (Alg) solution with $30 \%$ from polymer weight before coating and crosslinking steps.

\section{Coating of PCL NFs with Alg using casting method} and crosslinking with $\mathrm{CaCl} 2$

Three $\mathrm{g}$ of alginate were dissolved in $100 \mathrm{~mL}$ DI (deionized) water. Afterward, PCL NFs were coated using casting method by Alg solutions and were left to dry in vacuum oven. Coated sheets were crosslinked by soaking in $2 \%$ Calcium chloride solution for 2 minutes and then dried in vacuum oven.

\author{
Final scaffolds \\ * S1: PCL NFs coated with Alg. \\ - S2: PCL NFs coated with Alg and crosslinked with \\ $\mathrm{CaCl}_{2}$. \\ - S3: PCL NFs coated with Alg (with n-HA) and \\ crosslinked with $\mathrm{CaCl}_{2}$.
}

\section{Isolation and characterization of ADSC}

Six male Sprague-Dawley rats of 200-250 g weight (5-6 months of age) were used for stem cell isolation as described by Sun et al. (23). ADSC were isolated from the abdominal fats of healthy rats. Adipose tissue was washed extensively and minced into pieces; $0.1 \%$ type I collagenase was used for tissue digestion for 1 hour at $37{ }^{\circ} \mathrm{C}$. DMEM/ F12 with $10 \%$ FBS was added to neutralize collagenase activity. Cells were incubated in a $5 \% \mathrm{CO}_{2}$ incubator at $37{ }^{\circ} \mathrm{C}$. The medium was changed every $2-3$ days. Cell cultures were maintained until they reached $80 \%$ confluence and were examined daily using an inverted microscope (Olympus, CKX41SF, Tokyo, Japan).

For characterization, ADSC at the third passage were trypsinized, washed with phosphate buffered saline (PBS) and incubated with antibodies against CD34, CD29 and CD90 (1:100) for 30 minutes at $4{ }^{\circ} \mathrm{C}$. Flow cytometry detection was performed using a BD Accuri C6 flow cytometer (BD Biosciences, USA).

\section{Seeding of ADSCs on scaffolds}

Scaffold samples $\left(5 \times 5 \mathrm{~mm}^{2}\right)$ were sterilized by using $70 \%$ ethanol and ultraviolet light, and then kept in culture media overnight, then replaced with fresh media. Scaffolds were placed in 6-well plates with one scaffold per well. ADSC were seeded onto scaffolds at a density of $4 \times 10^{5}$ per sample by application dropwise on top of the scaffolds, cultured in complete media and incubated in an atmosphere of $5 \%$ $\mathrm{CO}_{2}$ at $37^{\circ} \mathrm{C}$. The media were changed every second day. After 7 days the media were changed into osteogenic media and changed every 3 days thereafter. A diagram showing the study steps is shown in Figure $S 1$ in the supplementary materials.

\section{ARS staining}

To assess calcium deposition, ARS staining was carried out. The cell supernatant was discarded, and cells were washed twice with PBS with $\mathrm{Ca} / \mathrm{Mg}$ then fixed with neutral 
buffered formalin (10\%) for 30 minutes and washed with PBS. ARS (40 mM in deionized water, $\mathrm{pH} 4.2$ ) was added to the cells and incubated for 45 minutes in the dark. ADSCs were washed four times and then PBS was added. For quantification, the stained cells were dissolved in $10 \%$ acetic acid and quantified using microplate reader (Biotek, ELx800).

\section{Reverse transcription and real time quantitative polymerase chain reaction (RT-qPCR)}

Total RNA was isolated using RNeasy Mini Kit (QIAGEN, Hilden, Germany) according to the manufacturer's instruction. Total RNA $(2 \mu \mathrm{g})$ was reverse transcribed into single-stranded cDNA using RevertAid First Strand cDNA Synthesis Kit (Thermo scientific, MA, USA). Primer from Vivantis Technologies Sdn. Bhd. (Shah Alam, Malaysia) was used at a final concentration of 10 pmol. The cDNA was amplified with the forward and reverse primer by RTqPCR. The primer sequence for alkaline phosphatase (ALP) was:

Forward 5'-GACAAGTTCCCCTTTGTGGC-3';

Reverse 5'-CCTTCACGCCACACAAGTAG-3'.

Real-time qPCR reaction was carried out in $20 \mu \mathrm{L}$ volume including $10 \mu \mathrm{L}$ of Maxima SYBR Green/ROX qPCR Master Mix (2×) (Thermo scientific, MA, USA), $2 \mu \mathrm{L}$ of forward and reverse primers ( $1 \mu \mathrm{L}$ for each), $1 \mu \mathrm{L}$ of cDNA, and $7 \mu \mathrm{L}$ of RNase-free water.

Reaction conditions were carried out with the following cycling stages in real-time thermal cycler (Thermo Fisher scientific, Vantaa, Finland): Initial denaturation at $95^{\circ} \mathrm{C}$ for 10 minutes, 40 cycles of denaturation at $95^{\circ} \mathrm{C}$ for 15 seconds, annealing at $60^{\circ} \mathrm{C}$ for 30 seconds and extension at $72{ }^{\circ} \mathrm{C}$ for 30 seconds. Glyceraldehyde- 3 phosphate dehydrogenase (GAP-DH) from Vivantis Technologies Sdn. Bhd. (Shah Alam, Malaysia) was used as a house-keeping gene with a sequence of:

Forward 5'-TTGTGCAGTGCCAGCCTCGT-3';

Reverse 5'-TGCCGTTGAACTTGCCGTGG-3'.

\section{Study design}

This is an experimental, randomized controlled study. This study consisted of five groups:

* Group 1 (negative control group): ADSCs were cultured with complete culture media.

* Group 2 (positive control group): ADSCs were cultured with osteogenic differentiation media.
* Group 3 (S1): ADSCs were seeded onto scaffold 1 (S1) (PCL/Alg) and cultured with osteogenic differentiation media.

* Group 4 (S2): ADSCs were seeded onto scaffold 2 (S2) (PCL/Alg/Ca) and cultured with osteogenic differentiation media.

* Group 5 (S3): ADSCs were seeded onto scaffold 3 (S3) (PCL/Alg/Ca/n-HA) and cultured with osteogenic differentiation media.

ADSCs were cultured in 6-well plates in triplicates for each group with density of $4 \times 10^{5}$ cells/well. Cell cultures were examined daily and assessed at 7, 14 and 21 days for:

(I) Mineralization using ARS staining.

(II) $A L P$ gene expression using RT-qPCR.

\section{Statistical analysis}

Data was presented as mean \pm standard deviation and was analyzed using Statistical Package for Social Science software computer program version 23 (SPSS Inc., Chicago, IL, USA). One way and two-way analysis of variance (ANOVA) and Tukey were used for comparing data. $\mathrm{P}$ value less than 0.05 was considered statistically significant.

\section{Results}

\section{Isolation and characterization of ADSCs}

MSCs were characterized by spindle, fibroblast-like morphology and adherence to plastic walls as mentioned in our previous study (24). Analysis of ADSCs surface marker expression by flow cytometry revealed that ADSCs were positive for the mesenchymal markers CD29 and CD90 and were negative for the hematopoietic marker CD34. Fluorescence-activated cell sorting (FACS) histograms are presented in Figure 1.

\section{ARS staining results}

Figures 2-4 show the ARS staining photomicrographs at 7,14 and 21 days respectively. Calcium deposits appear as aggregation of red stain. Figure $5 A$ shows the bar graph representing the one-way ANOVA results for ARS. Scaffold 3 group (S3) (PCL/Alg/Ca/n-HA) showed significantly higher mineralization levels than all groups through the whole experiment. Scaffold 1 (S1) (PCL/Alg) and scaffold 2 (S2) (PCL/Alg/Ca) groups showed almost equal values with no significant difference at all periods. Tables 1 and 2 show 

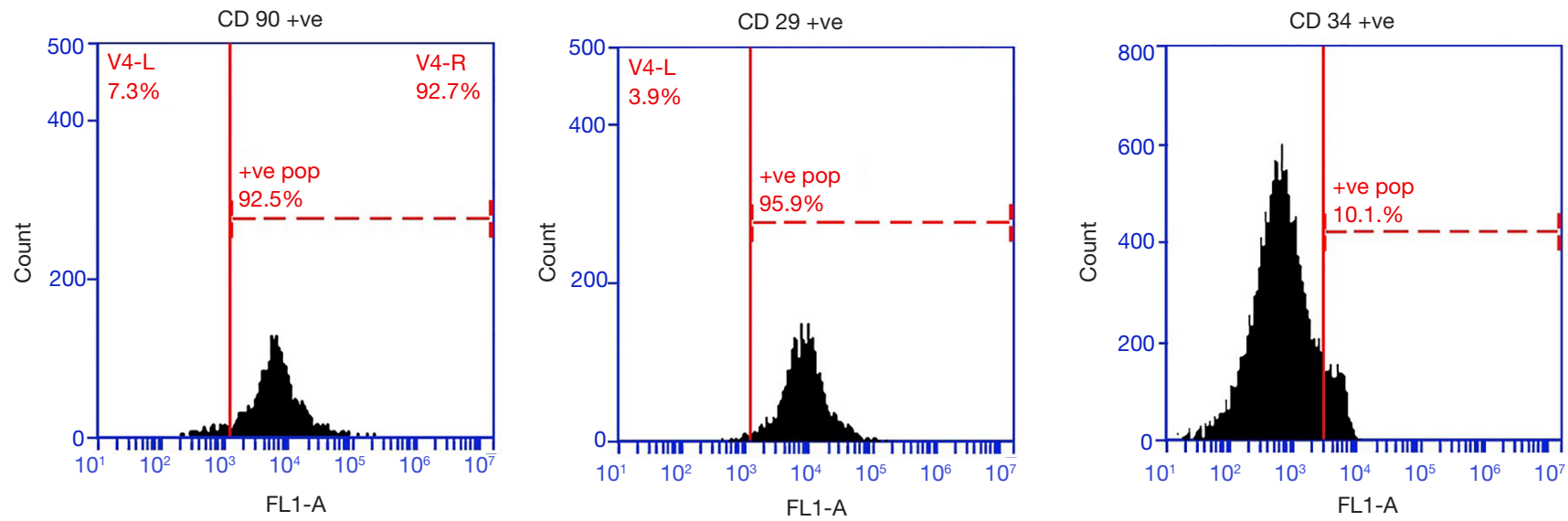

Figure 1 Flowcytometry histogram of ADSCs.
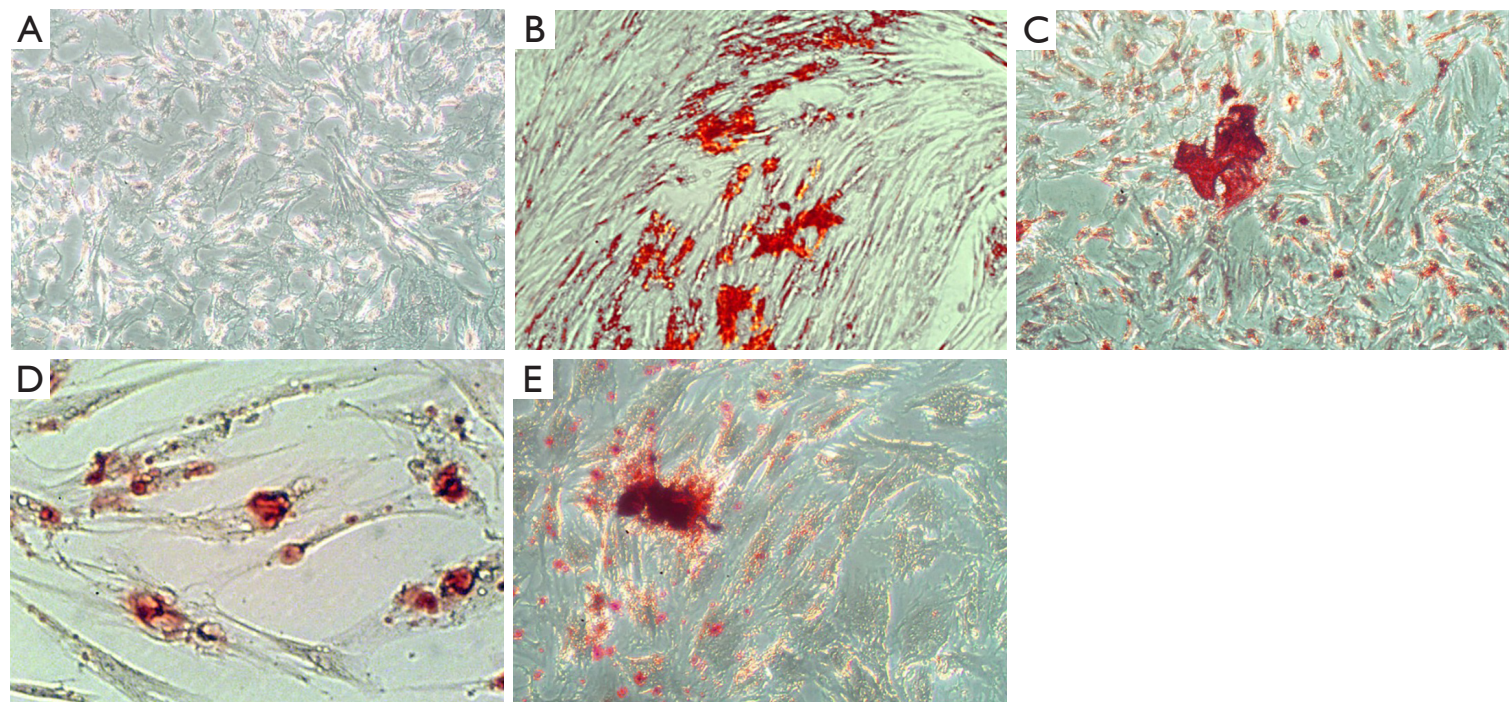

Figure 2 Photomicrograph showing Alizarin red S staining at day 7. (A) Negative control group (ADSCs + culture media) (4x). (B) Positive control group (ADSCs + osteogenic media) (4×). (C) S1 group (PCL/Alg) (4×). (D) S2 group (PCL/Alg/Ca) (10×). (E) S3 group (PCL/Alg/ $\mathrm{Ca} / \mathrm{n}-\mathrm{HA})(10 \times)$.

the two-way ANOVA results for ARS. The marginal means of ARS staining for the different groups, as shown in Table 1, confirm that $\mathrm{S} 3$ group had the highest mineralization values with significant difference than other groups.

According to the bar graph in Figure $5 A$, mineralization levels increased progressively with time, where the control positive group and all scaffolds showed significantly higher values at days 14 and 21 than day 7. Moreover, the mineralization value for each group increased at day 21 when compared to day 14 but with no significant difference except for S1 group which was significantly higher at day
21 than day 14. Table 2 shows the marginal means of ARS at the different time periods and it reflects a time-dependent increase in calcium deposition through days 7, 14 and 21 with significant difference.

\section{$R T-q P C R$ results}

Gene expression of the osteogenic marker ALP as determined by RT-qPCR showed increased expression at day 7 with significantly higher values for the three scaffolds' groups than the negative and positive control groups. The 

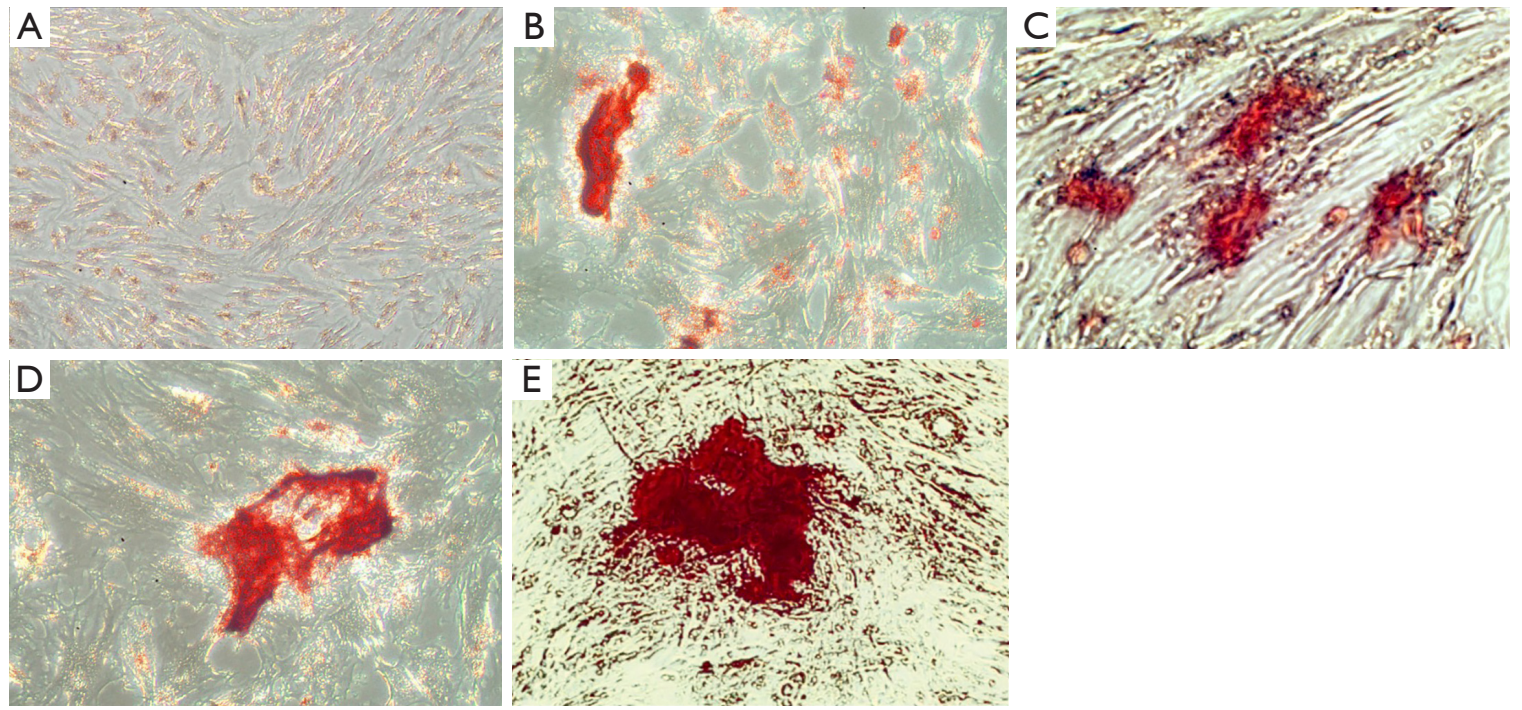

Figure 3 Photomicrograph showing Alizarin red S staining at day 14. (A) Negative control group (ADSCs + culture media) (4x). (B) Positive control group (ADSCs + osteogenic media) (10x). (C) S1 group (PCL/Alg) (40×). (D) S2 group (PCL/Alg/Ca) (10×). (E) S3 group (PCL/ $\mathrm{Alg} / \mathrm{Ca} / \mathrm{n}-\mathrm{HA})(40 \times)$. ADSC, adipose derived stem cell; PCL/Alg, polycaprolactone/alginate; n-HA, nano-hydroxyapatite.
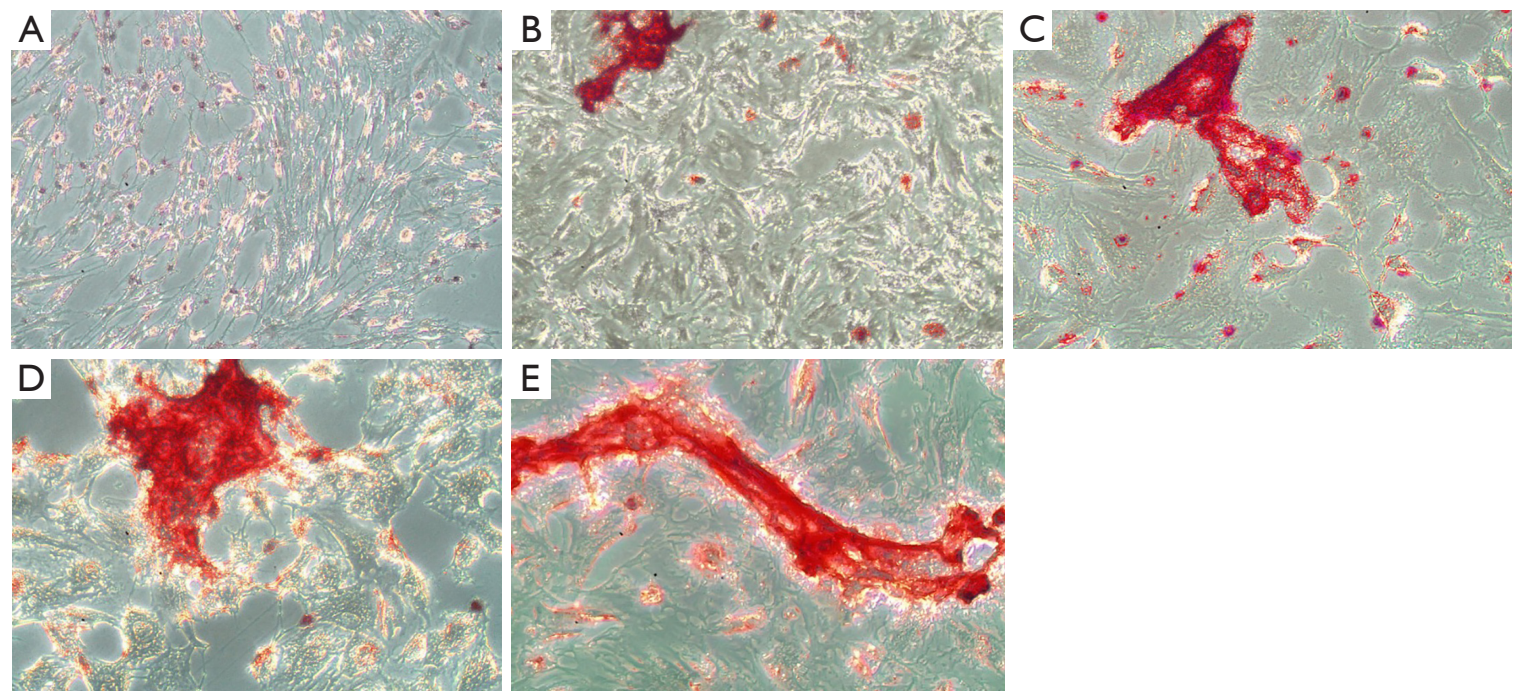

Figure 4 Photomicrograph showing Alizarin red S staining at day 21. (A) Negative control group (ADSCs + culture media) (4x). (B) Positive control group (ADSCs + osteogenic media) (4x). (C) S1 group (PCL/Alg) (10x). (D) S2 group (PCL/Alg/Ca) (10x). (E) S3 group (PCL/Alg/ $\mathrm{Ca} / \mathrm{n}-\mathrm{HA})(10 \times)$. ADSC, adipose derived stem cell; PCL/Alg, polycaprolactone/alginate; n-HA, nano-hydroxyapatite.

second scaffold (S2) (PCL/Alg/Ca) showed significantly higher gene expression than all groups at day 7 .

At days 14 and 21, gene expression of all groups sharply declined with significant difference than the negative control group and than their counterparts at day 7 . Fluctuation in gene expression levels was detected where all values declined sharply at day 14 and then slightly increased at day 21. Figure $5 B$ shows the bar graph representing the one-way ANOVA results for $A L P$ gene expression.

Tables 1 and 2 show the 2-way ANOVA results for ALP gene expression. The marginal means of ALP relative gene expression for the different groups through the experiment, 

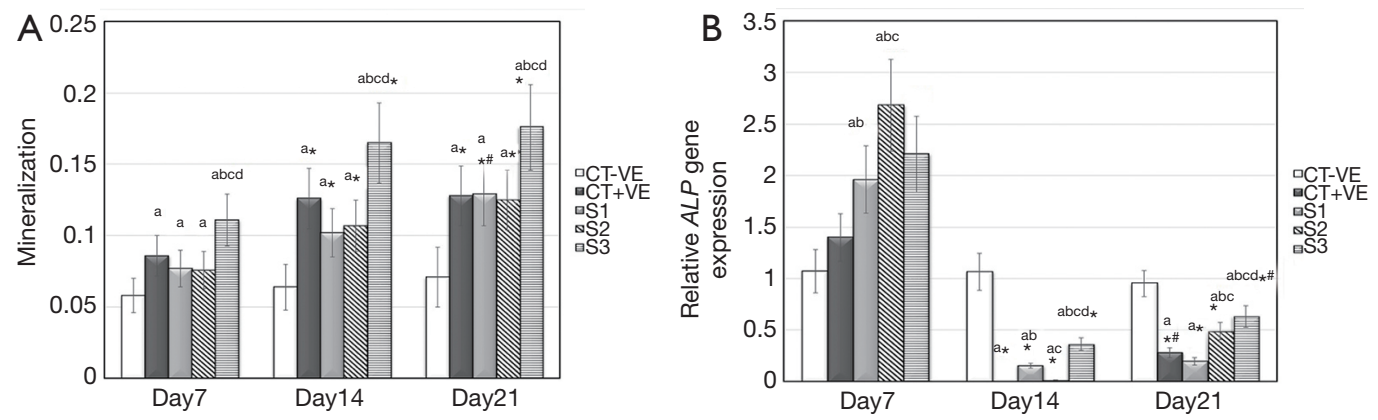

Figure 5 Bar graphs showing one-way ANOVA analysis. (A) Bar graph showing one-way ANOVA analysis for ARS mean values. (B) Bar graph showing one-way ANOVA analysis for $A L P$ gene expression mean values. a , significance $v s$. -ve control group; ${ }^{\text {, }}$, significance $v s$. +ve control group; ${ }^{c}$, significance $v s$. S1 group; ${ }^{\text {d }}$, significance $v s$. S2 group within each time period; *, significance $v s$. day 7 group; ${ }^{*}$, significance vs. day 14 group within each group. $\mathrm{P}<0.05$. ANOVA, analysis of variance; ARS, Alizarin red S; ALP, alkaline phosphatase.

Table 1 Marginal means of ARS and ALP values of different groups and their effect over time (two-way ANOVA)

\begin{tabular}{|c|c|c|c|c|c|c|}
\hline & -VE CT & +VE CT & S1 (PCL/Alg) & S2 (PCL/Alg/Ca) & S3 (PCL/Alg/Ca/n-HA) & $P$ \\
\hline ARS & $0.0643 \pm 0.0163$ & $0.1133 \pm 0.0210^{a}$ & $0.1027 \pm 0.0187^{a}$ & $0.1027 \pm 0.0183^{a}$ & $0.1507 \pm 0.0253^{a, b, c, d}$ & $<0.001$ \\
\hline ALP & $1.031 \pm 0.171$ & $0.563 \pm 0.094^{a}$ & $0.772 \pm 0.129^{\mathrm{a}, \mathrm{b}}$ & $1.063 \pm 0.177^{\mathrm{b}, \mathrm{c}}$ & $1.068 \pm 0.178^{\mathrm{b}, \mathrm{c}}$ & $<0.001$ \\
\hline
\end{tabular}

${ }^{a}$, significance vs. -ve control group; ${ }^{b}$, significance vs. +ve control group; ${ }^{c}$, significance vs. S1 group; ${ }^{d}$, significance vs. S2 group within each time period. $\mathrm{P}$, probability $\mathrm{P}<0.05$. ANOVA, analysis of variance; ARS, Alizarin red S; ALP, alkaline phosphatase; PCL/Alg, polycaprolactone/alginate; n-HA, nano-hydroxyapatite.

Table 2 Marginal means of ARS and ALP values of different time periods and their effect over groups (two-way ANOVA)

\begin{tabular}{ccccc}
\hline & Day 7 & Day 14 & Day 21 & $P$ \\
\hline ARS & $0.0816 \pm 0.014$ & $0.1128 \pm 0.02^{\star}$ & $0.1258 \pm 0.0258^{\star *}$ & $<0.001$ \\
ALP & $1.865 \pm 0.317$ & $0.322 \pm 0.054^{*}$ & $0.511 \pm 0.079^{* \#}$ & $<0.001$ \\
\hline
\end{tabular}

*, significance vs. day 7 group; ", significance vs. day 14 group within each group. $\mathrm{P}$, probability $\mathrm{P}<0.05$. ANOVA, analysis of variance; ARS, Alizarin red S; ALP, alkaline phosphatase.

as shown in Table 1, show that gene expression levels for $\mathrm{S} 2$ (PCL/Alg/Ca) and S3 (PCL/Alg/Ca/n-HA) groups were significantly higher than the positive control and S1 (PCL/ $\mathrm{Alg}$ ) groups but insignificantly different from each other.

\section{Discussion}

Bone tissue engineering is a promising field that has experienced a huge growth in the last years aiming to induce bone regeneration by combining cells, biomaterials and morphological signals together (25). Different PCLbased scaffolds have been used to enhance osteogenic differentiation of different mesenchymal stem cells $(17,26,27)$. In this study we managed to test a novel PCL/
$\mathrm{Alg} / \mathrm{Ca} / \mathrm{n}-\mathrm{HA}$ scaffold regarding its effect on the osteogenic capacity of rat adipose mesenchymal stem cells and to compare it with other PCL-based scaffolds.

In this study, mineralization was detected by ARS staining where ARS has been widely used to stain calcium deposits in cell cultures (28) and to determine cell osteogenic differentiation levels (12). Mineralization values showed progressive increase starting from day 7 through days 14 and 21 . This is consistent with a study by Kim \& Kim (12) who compared between different concentrations of alginate added to the PCL scaffolds seeded with Mouse pre-osteoblast cells. The ARS staining values increased at day 14 than day 7 .

In the same context, another study detected elevated 
calcium deposition levels at day 21 using a PCL/HA/ $\mathrm{TZ}$ (thermoplastic zein) scaffold co-cultured with rabbit bone marrow stem cells (17). In a similar study testing the osteogenic differentiation of dental pulp stem cells (DPSCs) on alginate/HA based scaffolds, mineralization as detected by ARS at days 7, 14, 21 and 28 showed to be time dependent (29).

$\mathrm{S} 3$ group (PCL/Alg/Ca/n-HA) showed the highest values for mineralization showing that addition of $n-H A$ particles aided in osteogenic differentiation of ADSCs and consequently increased calcium deposition levels. This finding coincides with the results by Sancilio et al. who found that $\mathrm{n}$-HA reinforced alginate scaffolds and acted as an osteoconductive component in $\mathrm{Alg} / \mathrm{n}-\mathrm{HA}$ composite scaffolds (29).

ALP is a critical enzyme for bone mineralization (30) and is considered as a reliable marker for osteoblast differentiation (31). In this study the relative gene expression value for ALP for all tested scaffolds showed significant increase than the negative control group at day 7 then, it significantly decreased at days 14 and 21 . This is similar to a study by Chen et al. who detected increase in $A L P$ gene expression at days 4 and 7 and decrease thereafter at days 14 and 21 in a comparison between PCL and PCL/Hyaluronic acid/tricalcium phosphate scaffolds (32).

Similarly Liao et al. detected increased ALP activity at day 7 , as measured by p-nitrophenyl phosphate assay, and time dependent decrease at days 14 and 21 when seeding ADSCs on different PCL scaffolds (26). Jafary et al. stated that $A L P$ gene expression was upregulated at day 3 and then significantly decreased at day 7 when seeding rat adipose MSCs on collagen scaffold with immobilized ALP (30).

Similarly, in other studies for the osteogenic effect of 3-D Zr-Si scaffold and Alg/Ha scaffold on human ADSCs and DPSCs respectively, ALP activity showed a peak value at day 3 and then showed a time dependent decrease till day $21(29,33)$.

In consistence with the current study, some studies showed fluctuations in $A L P$ gene expression and ALP activity levels by time with different scaffolds and different types of MSCs (33-35). On the other hand, in contrast to our results, some authors detected a time dependent increase in ALP relative gene expression levels and ALP activity $(12,27,36,37)$.

In the present study, mineralization as detected by ARS stain increased with time through the experiment, while inversely, $A L P$ gene expression decreased significantly after day 7. This can be explained by knowing that osteogenesis has 3 major phases: the first is proliferation, the second is deposition of extracellular matrix and maturation, while the third is mineralization, with ALP being highly expressed early at the end of the first and during the second stages of osteogenesis (35).

Also Liao et al. confirmed that ALP is an early expression gene during osteogenic differentiation (26) and Jensen et al. stated that ALP enzyme is produced by active osteoblasts in the early induction of mineralization (37). This justifies the upregulation of ALP at day 7 and its decline thereafter at later stages of osteogenesis. This also justifies the increased mineralization levels detected by ARS through days 7, 14 and 21 where mineralization is the last stage of osteogenesis as fore mentioned.

As detected by the two-way ANOVA results, $A L P$ gene expression levels for S2 (PCL/Alg/Ca) and S3 (PCL/Alg/ $\mathrm{Ca} / \mathrm{n}-\mathrm{HA})$ groups were significantly higher than $\mathrm{S} 1$ group (PCL/Alg) and showed no significant difference in-between. This implies that crosslinking PCL and $\mathrm{Alg}$ with $\mathrm{CaCl}_{2}$ aided the osteoblastic differentiation process and $A L P$ gene expression. This agrees with Hernandez et al. who mentioned that crosslinking with calcium chloride enhances the stability and retention of alginate to PCL sheets (38). These results also indicated that incorporating n-HA in the scaffold efficiently enhanced osteogenesis.

\section{Conclusions}

This in vitro study showed ADSCs to be good candidates for bone tissue engineering. It also concluded that crosslinking with $\mathrm{CaCl}_{2}$ and adding n-HA particles to PCL/Alg nanoscaffolds efficiently improved the osteogenic differentiation ability of ADSCs and enhanced the mineralization process. So, PCL/Alg/Ca/n-HA nano-fibrous scaffold may hold a great promise in bone tissue engineering field and this requires further in vivo studies.

\section{Acknowledgments}

Funding: None.

\section{Footnote}

Conflicts of Interest: All authors have completed the ICMJE uniform disclosure form (available at http://dx.doi. org/10.21037/sci-2020-015). The authors have no conflicts of interest to declare. 
Ethical Statement: The authors are accountable for all aspects of the work in ensuring that questions related to the accuracy or integrity of any part of the work are appropriately investigated and resolved. All procedures involving animals were approved by the ethical committee of faculty of dentistry, Mansoura University with code number: A13091019, in compliance with Mansoura University guidelines for the care and use of animals.

Open Access Statement: This is an Open Access article distributed in accordance with the Creative Commons Attribution-NonCommercial-NoDerivs 4.0 International License (CC BY-NC-ND 4.0), which permits the noncommercial replication and distribution of the article with the strict proviso that no changes or edits are made and the original work is properly cited (including links to both the formal publication through the relevant DOI and the license). See: https://creativecommons.org/licenses/by-nc-nd/4.0/.

\section{References}

1. Guerado E, Caso E. Challenges of bone tissue engineering in orthopaedic patients. World J Orthop 2017;8:87.

2. Salgado AJ, Coutinho OP, Reis RL. Bone tissue engineering: state of the art and future trends. Macromol Biosci 2004;4:743-65.

3. Horwitz E, Le Blanc K, Dominici M, et al. Clarification of the nomenclature for MSC: The International Society for Cellular Therapy position statement. Cytotherapy 2005;7:393-5.

4. Orbay H, Tobita M, Mizuno H. Mesenchymal stem cells isolated from adipose and other tissues: basic biological properties and clinical applications. Stem Cells Int 2012;2012:461718.

5. Zuk PA. The adipose-derived stem cell: looking back and looking ahead. Mol Biol Cell 2010;21:1783-7.

6. Rios HF, Lin Z, Oh B, et al. Cell-and gene-based therapeutic strategies for periodontal regenerative medicine. J Periodontol 2011;82:1223-37.

7. Discher DE, Mooney DJ, Zandstra PW. Growth factors, matrices, and forces combine and control stem cells. Science 2009;324:1673-7.

8. Manjubala I, Scheler S, Bössert J, et al. Mineralisation of chitosan scaffolds with nano-apatite formation by double diffusion technique. Acta Biomater 2006;2:75-84.

9. Xue R, Qian Y, Li L, et al. Polycaprolactone nanofiber scaffold enhances the osteogenic differentiation potency of various human tissue-derived mesenchymal stem cells.
Stem Cell Res Ther 2017;8:148.

10. Kundu J, Shim JH, Jang J, et al. An additive manufacturing-based PCL-alginate-chondrocyte bioprinted scaffold for cartilage tissue engineering. J Tissue Eng Regen Med 2015;9:1286-97.

11. Lee KY, Mooney DJ. Alginate: properties and biomedical applications. Prog Polym Sci 2012;37:106-26.

12. Kim YB, Kim GH. PCL/alginate composite scaffolds for hard tissue engineering: fabrication, characterization, and cellular activities. ACS Comb Sci 2015;17:87-99.

13. Luo Y, Lode A, Sonntag F, et al. Well-ordered biphasic calcium phosphate-alginate scaffolds fabricated by multichannel 3D plotting under mild conditions. J Mater Chem B 2013;1:4088-98.

14. Venkatesan J, Bhatnagar I, Manivasagan P, et al. Alginate composites for bone tissue engineering: a review. Int $\mathrm{J}$ Biol Macromol 2015;72:269-81.

15. Jeon YJ, Shahidi F, KIM SK. Preparation of chitin and chitosan oligomers and their applications in physiological functional foods. Food Rev Int 2000;16:159-76.

16. Gallardo-López A, Domínguez-Rodríguez A, Estournès $\mathrm{C}$, et al. Plastic deformation of dense nanocrystalline yttrium oxide at elevated temperatures. J Eur Ceram Soc 2012;32:3115-21.

17. Salerno A, Oliviero M, Di Maio E, et al. Design of novel three-phase PCL/TZ-HA biomaterials for use in bone regeneration applications. J Mater Sci Mater Med 2010;21:2569-81.

18. Turco G, Marsich E, Bellomo F, et al. Alginate/ hydroxyapatite biocomposite for bone ingrowth: a trabecular structure with high and isotropic connectivity. Biomacromolecules 2009;10:1575-83.

19. Tohamy KM, Mabrouk M, Soliman IE, et al. Novel alginate/hydroxyethyl cellulose/hydroxyapatite composite scaffold for bone regeneration: In vitro cell viability and proliferation of human mesenchymal stem cells. Int J Biol Macromol 2018;112:448-60.

20. Kim JW, Shin KH, Koh YH, et al. Production of poly ( $\varepsilon$-caprolactone)/hydroxyapatite composite scaffolds with a tailored macro/micro-porous structure, high mechanical properties, and excellent bioactivity. Materials 2017;10:1123.

21. Yahia S, Khalil IA, El-Sherbiny IM. Sandwich-Like Nanofibrous Scaffolds for Bone Tissue Regeneration. ACS Appl Mater Interfaces 2019;11:28610-20.

22. El-Sherbiny IM, Yahia S, Messiery MA, et al. Preparation and physicochemical characterization of new nanocomposites based on $\beta$-type chitosan and nano- 
hydroxyapatite as potential bone substitute materials. Int J Polym Mater 2014;63:213-20.

23. Sun J, Liu WH, Deng FM, et al. Differentiation of rat adipose-derived mesenchymal stem cells into corneal-like epithelial cells driven by PAX6. Exp Ther Med 2018;15:1424-32.

24. Hany E, Sobh MA, ElKhier MTA, et al. The Effect of Different Routes of Injection of Bone Marrow Mesenchymal Stem Cells on Parotid Glands of Rats Receiving Cisplatin: A Comparative Study. Int J Stem Cells 2017;10:169.

25. Amini AR, Laurencin CT, Nukavarapu SP. Bone tissue engineering: recent advances and challenges. Crit Rev Biomed Eng 2012;40:363-408.

26. Liao HT, Lee MY, Tsai WW, et al. Osteogenesis of adipose-derived stem cells on polycaprolactone$\beta$-tricalcium phosphate scaffold fabricated via selective laser sintering and surface coating with collagen type I. J Tissue Eng Regen Med 2016;10:E337-53.

27. Li J, Chen M, Wei X, et al. Evaluation of 3D-printed polycaprolactone scaffolds coated with freeze-dried platelet-rich plasma for bone regeneration. Materials 2017;10:831.

28. Gregory CA, Gunn WG, Peister A, et al. An Alizarin redbased assay of mineralization by adherent cells in culture: comparison with cetylpyridinium chloride extraction. Anal Biochem 2004;329:77-84.

29. Sancilio S, Gallorini M, Di Nisio C, et al. Alginate/ hydroxyapatite-based nanocomposite scaffolds for bone tissue engineering improve dental pulp biomineralization and differentiation. Stem Cells Int 2018;2018:9643721.

30. Jafary F, Hanachi P, Gorjipour K. Osteoblast Differentiation on Collagen Scaffold with Immobilized Alkaline Phosphatase. Int J Organ Transplant Med 2017;8:195-202.

doi: $10.21037 /$ sci-2020-015

Cite this article as: Hany E, Yahia S, Elsherbeny MF, Salama NM, Ateia IM, Abou El-Khier NT, El-Sherbiny I, Abou Elkhier MT. Evaluation of the osteogenic potential of rat adipose-derived stem cells with different polycaprolactone/ alginate-based nanofibrous scaffolds: an in vitro study. Stem Cell Investig 2020;7:14.
31. Hashemibeni B, Jafary F, Esmaeil N, et al. Comparison of Phenotypic Characterization between Differentiated Osteoblasts from Stem Cells and Calvaria Osteoblasts In vitro. Int J Prev Med 2013;4:180-6.

32. Chen M, Le DQ, Kjems J, et al. Improvement of Distribution and Osteogenic Differentiation of Human Mesenchymal Stem Cells by Hyaluronic Acid and betaTricalcium Phosphate-Coated Polymeric Scaffold In Vitro. Biores Open Access 2015;4:363-73.

33. Koroleva A, Deiwick A, Nguyen A, et al. Osteogenic differentiation of human mesenchymal stem cells in 3-D $\mathrm{Zr}$-Si organic-inorganic scaffolds produced by two-photon polymerization technique. PLoS One 2015;10:e0118164.

34. Hammerl A, Diaz Cano CE, De-Juan-Pardo EM, et al. A Growth Factor-Free Co-Culture System of Osteoblasts and Peripheral Blood Mononuclear Cells for the Evaluation of the Osteogenesis Potential of MeltElectrowritten Polycaprolactone Scaffolds. Int J Mol Sci 2019;20:1068.

35. Zur Nieden NI, Kempka G, Ahr HJ. In vitro differentiation of embryonic stem cells into mineralized osteoblasts. Differentiation 2003;71:18-27.

36. Kim Y, Kim G. Collagen/alginate scaffolds comprising core (PCL)-shell (collagen/alginate) struts for hard tissue regeneration: fabrication, characterisation, and cellular activities. J Mater Chem B 2013;1:3185-94.

37. Jensen J, Kraft DCE, Lysdahl H, et al. Functionalization of polycaprolactone scaffolds with hyaluronic acid and $\beta$-TCP facilitates migration and osteogenic differentiation of human dental pulp stem cells in vitro. Tissue Eng Part A 2015;21:729-39.

38. Hernandez I, Kumar A, Joddar B. A bioactive hydrogel and $3 \mathrm{~d}$ printed polycaprolactone system for bone tissue engineering. Gels 2017;3:26. 


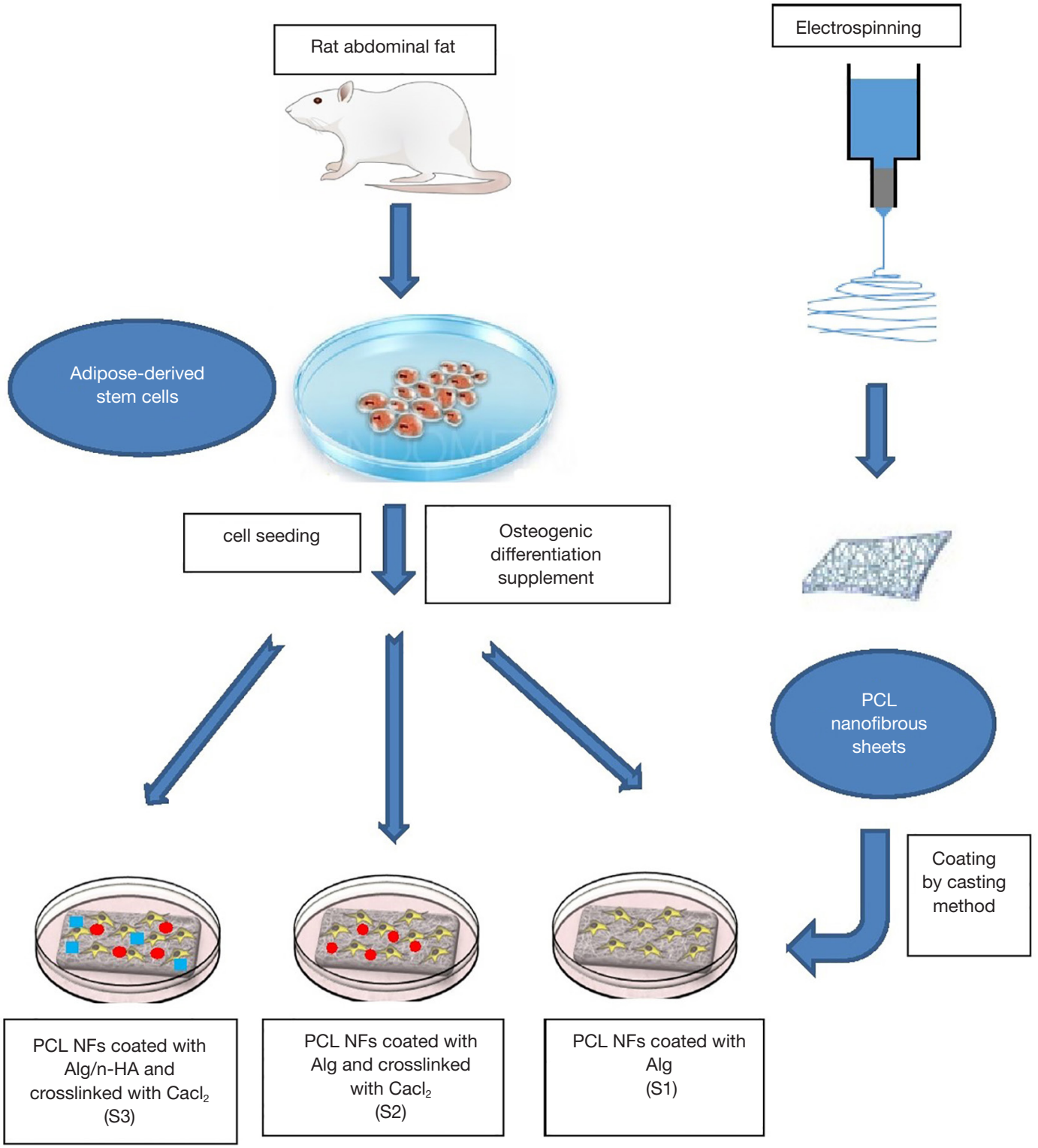

Figure S1 Graphical abstract. 